\title{
Charge Selection Algorithms for Maximizing Sensor Network Life with UAV-Based Limited Wireless Recharging
}

\author{
Jennifer Johnson ${ }^{\# 1}$, Elizabeth Basha ${ }^{\# 2}$, Carrick Detweiler ${ }^{* 3}$ \\ \# Department of Electrical and Computer Engineering, The University of the Pacific \\ 3601 Pacific Avenue, Stockton, California 95211, USA \\ * Computer Science and Engineering, University of Nebraska-Lincoln \\ 220 Schorr Center, Lincoln, NE, 68588, USA \\ ${ }^{1}$ j_johnson31@u.pacific.edu \\ 2 ebasha@pacific.edu \\ ${ }^{3}$ carrickecse.unl. edu
}

\begin{abstract}
Monitoring bridges with wireless sensor networks aids in detecting failures early, but faces power challenges in ensuring reasonable network lifetimes. Recharging select nodes with Unmanned Aerial Vehicles (UAVs) provides a solution that currently can recharge a single node. However, questions arise on the effectiveness of a limited recharging system, the appropriate node to recharge, and the best sink selection algorithm for improving network lifetime given a limited recharging system. This paper simulates such a network in order to answer those questions. It explores five different sink positioning algorithms to find which provides the longest network lifetime with the added capability of limited recharging. For a range of network sizes, our results show that network lifetime improves by over $350 \%$ when recharging a single node in the network, the best node to recharge is the one with the lowest power level, and that either the Greedy Heuristic or LP sink selection algorithms perform equally well.
\end{abstract}

\section{INTRODUCTION}

Failing bridges have become a heated issue given the recent collapses of the Minneapolis I35 bridge in 2007 [10] and the California Bay Bridge in 2009 [17]. Work in structural health monitoring attempts to find methods of automating the inspections and detections. The process to date involves instrumenting a bridge with sensors that monitor vibrations [6], [12], [13], [18]. With this instrumentation, structural engineers can use techniques such as modal analysis [14] to analyze the health of the bridge. This and similar techniques require highfrequency data collection at multiple locations along the bridge at times when vehicles are driving over the bridge.

Deploying power and communication infrastructure for the sensors is a significant cost in monitoring bridges. Wireless sensor networks, on the other hand, could be easily and quickly deployed, programmed to wake when traffic is detected to monitor vibrations, and programmed to wirelessly communicate the results back to a data collection point. This removes the cost associated with cables for communication; however, powering this bridge-monitoring wireless sensor network still poses a challenge. Under bridges, solar charging is unavailable and adding wires to the top or edge of the bridge removes one of the main benefit of using a wireless sensor network.

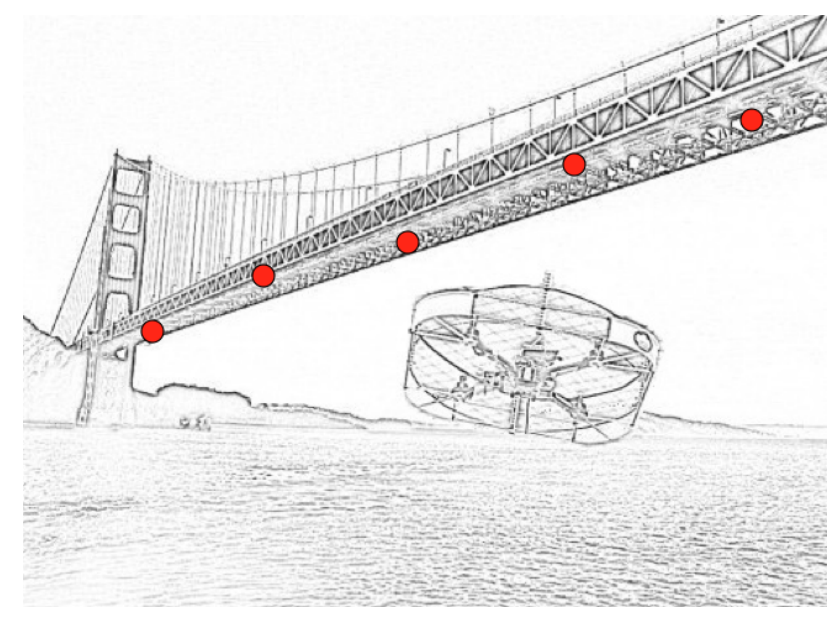

Fig. 1. Scenario showing UAV going to recharge sensors on a bridge.

To solve this energy problem, we consider utilizing an unmanned aerial vehicle (UAV) system to recharge a select number of nodes through wireless power transfer (see Figure 1), a system we previously described [8]. This wireless power transfer system enables the UAV to travel to remote and hard to reach locations to recharge sensor nodes. At this time, however, flight time constraints limit the UAV to charging only one or two nodes per flight. With a system that can selectively charge only a subset of the overall network, we need to consider which subset to recharge and how to effectively utilize the network between recharging.

This paper explores which node to recharge to optimize the overall lifetime of the network. Other research on optimizing network life focuses on decreasing power consumption and increasing energy efficiency through the manipulation of MAC protocols, routing procedures and node versatility [7], [11], [20], [23], [25]-[27]. We also examine a subset of these methods within our novel recharging framework to determine the impact of selective charging on these algorithms.

The paper asks three questions: (1) does recharging a single node effectively increase the network lifetime, (2) which 
improves network lifetime the most - recharging the sink or recharging the node with the lowest energy level, and (3) which sink algorithms and heuristics best utilize the recharging system to increase network lifetime. To explore the first two questions, we define a battery capacity level for all nodes, power discharge equations based on communication (as that will require the most power), and recharging of only a single node per iteration. For the third question, we focus on four main sink positioning methods: static sink positioning, random sink movement, controlled sink movement, and dynamic sink movement (using both greedy and linear programming methods). These algorithms are taken and applied to an ideal network definition with the added capability of recharging a single node.

To explore and understand our questions, we develop a system simulator in Matlab that models the topology, routing, and recharging systems. In this simulator, all sensors are homogeneous and self aware of their position in the network. Topologies are grids that vary in both $\mathrm{x}$ and $\mathrm{y}$ dimensions with nodes equally spaced (similar to an under-bridge installation). Routing is Euclidean shortest path routing to the sink without consideration for packet loss. In all experiments, the metric of note is time to first failure at which point we declare the network dead.

Our results indicate that limited recharging does improve network lifetime over $350 \%$ compared to no recharging, especially if the node recharged has the lowest energy, although recharging the sink does help. In choosing a sink selection algorithm in a system with limited recharging of the lowest node, the Greedy Heuristic Sink and Linear Programming (LP) Sink algorithms provided the longest network lifetimes. As the Greedy Heuristic requires less computation, it performs the best overall.

The rest of this paper is organized as follows. Section II describes the related literature. Section III defines our network system, while Section IV outlines the sink selection algorithms. Section V introduces our simulation environment and describes the results and Section VI concludes the paper.

\section{RELATED WORK}

Existing research extensively studied sink algorithms and mobility patterns to reduce communications costs in wireless sensor networks. Some research looked into creating more efficient and cost effective hardware communication modules [27]. Medium access control protocols have also been heavily discussed and researched as an avenue of boosting network energy efficiency [25], [27].

In addition, a large portion of network communication research has been spent in data routing strategies such as multiple different dissemination protocols, tree structured protocols and power and sink aware sensor protocols [2], [3], [7], [11].

Moving the sink via sink selection algorithms provides four categories of algorithms: static [15], [21], random [5], controlled sink algorithms [4], [9], [15], [16], [22], [24], and dynamic sink algorithms [4]. Additional improvements include making the sink mobile [2]. This type of movement can refer

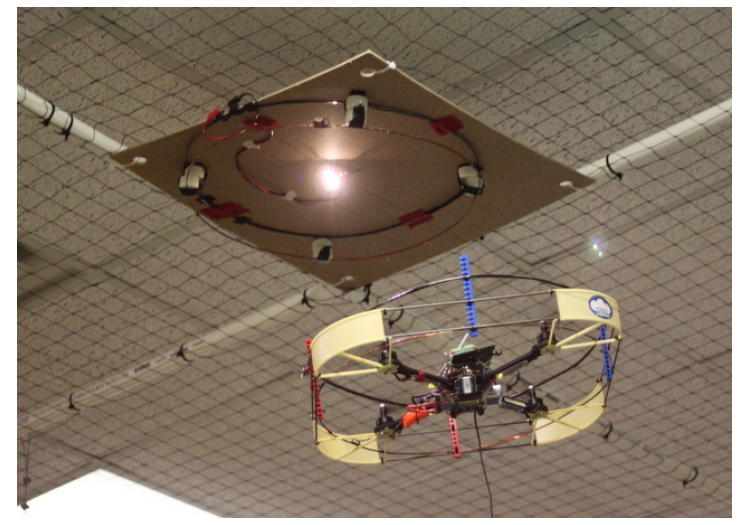

Fig. 2. UAV wirelessly powering a sensor with a LED

to either a sink obtaining control of an otherwise normal sensor node, or the sink physically moving itself from one position to the next [5], [11], [19]. We selected a representative set covering the major areas to examine in regards to our set of recharging questions; Section IV describes them in detail.

Our work adapts these energy efficient sink selection strategies to the novel problem of sink selection in networks that can selectively recharge specific nodes. To the best of our knowledge, no prior work explores scheduled recharging of selected nodes.

\section{Network AND ReCharging Models}

We now define our network model including the overall topology, data and communication rates, and routing scheme. In addition to the network model, we describe the recharging system and the model used for it. The network model is simplified to enable detailed analysis of the sink selection algorithm and recharging, which are the focus of this paper. Future work will examine implementation of these algorithms on real sensor network hardware.

\section{A. Network Model}

The network model assumes all sensor nodes are homogeneous, each with the capability of being the sink. We examine rectangular grid topologies of varying sizes with equal node distribution and assume each node is geographically localized.

For communication, we assume that data and packet transmission rates are regular and constant. Each node can communicate bi-directionally only with its direct neighbors when not operating as the sink node. In our simulation experiments, we assume that each transmitted and received packet of information is done so without error. We assume that each transmission uses 0.05 battery units and that each node has a capacity of 50 units. This means that each node can transmit 1000 times before needing recharging. Future work will examine implementations on a deployed in-situ network.

For routing, we use a multi-hop, shortest path routing algorithm to the sink. Each node forwards its packet to the sink during one time interval. At the start of the next time interval the sink will move if the implemented algorithm instructs it to. The time intervals continue until the network lifetime is 
reached, which we define here to be the time until the first node fails or the system reaches 200 iterations.

\section{B. Recharging Models}

In prior work we developed a UAV that can travel to remote locations to wirelessly recharge sensor nodes from a few feet away [8]. Figure 2 shows the UAV wirelessly transferring energy to a sensor node that is lighting a LED. We demonstrated that this system is able to transfer over $5 \mathrm{~W}$ in flight and current prototypes can transfer up to $15 \mathrm{~W}$. The main limitation of the system is that the UAV can only carry a limited amount of energy and is therefore limited to recharging only one or two sensor nodes per flight. In the simulations we assume that the UAV can recharge a single node and that it is able to charge this node with an amount of energy proportional to the size of the grid. As the grid grows larger, the number of data packets increases, creating a larger drain on the network energy. To account for this inequality of energy usage, the amount of power recharged in one node is higher when the grid size is larger. In practice, this increased charging can be achieved by sending out larger or multiple UAVs as the grid size grows. Details of the simulation are presented in Section V.

\section{Sink Selection Algorithms}

Sink algorithms are generally divided based on how the sink moves: static, random, controlled, and dynamic. We chose algorithms representative of these categories, with two in the controlled section, in order to provide insight into which category and type of selection algorithms performs best with limited recharging. This section describes the five sink selection algorithms.

\section{A. Static Sink Positioning}

The Static Sink algorithm fixes the sink at a stationary location throughout the lifetime of the sensor network. In determining which node to fix as the sink, we examine early sensor network research that concentrated on finding the optimal static position of the sink in the network [21]. We utilize the work of Luo et al. [15] which mathematically proved the best positioning of a static sink is in the center of the network and therefore fix our static sink in the center.

\section{B. Random Sink Movement}

Our second algorithm, Random Sink, moves the sink according to a random function and repositions it at every time interval. This category of sink selection usually focuses on a mobile sink moving randomly through the system (see [5] as an example), demonstrating improved performance over static sinks; as the sink is not mobile in our network, a random function choosing a new sink best captures this category.

\section{Controlled Sink Movement: Circles}

The Circles Sink algorithm controls the sink movement instead of using random motion. It is based on work by Luo et al. [15], which mathematically showed that programming the sink to move on the perimeter of the network improves the efficiency of power usage over moving the sink in a circular pattern closer to the center of the network. In their multi-hop network, moving the sink around the whole of the perimeter equalized the load of the communication traffic through each sensor, thus leading to an increase in the network lifetime. Our algorithm follows this perimeter walk approach and moves the sink to the next node around the perimeter.

\section{Controlled Sink Movement: LP}

A different approach to controlled sink movement utilizes linear programming model to determine the next sink, providing our fourth algorithm, LP Sink. This algorithm draws inspiration from research done by Wang et al. [24] which explores ways of creating an optimal controlled sink mobility algorithm in order to increase network lifetime instead of optimizing energy usage.

Using a gridded, multi-hop network with limited battery capacity, the paper formulated a linear programming problem to maximize the network lifetime subject to the constraint that the cost of a single node transmitting and receiving packets of data is less than the initial energy of the node. Equations 1 through 3 outline this structure.

\section{Max Network Lifetime:}

$$
\sum_{\forall k \in N} t_{k}
$$

Subject to:

$$
\begin{aligned}
& \sum_{\forall k \in N} c_{i}^{k} t_{k} \leq e_{0} \\
& t_{k} \geq \forall k \in N
\end{aligned}
$$

Where:

- $\mathbf{N}$ is the set of all nodes in the grid.

- $\mathbf{k}$ represents the current sink.

- $\mathbf{i}$ represents a general node in the grid.

- $t_{k}$ represents the sojourn time spent at each sink.

- $c_{i}^{k}$ represents the cost of one node sending and receiving data in one time interval when the sink is at position $\mathrm{k}$.

The paper defined the network lifetime as the sum of the total sink sojourn times before any single node fails. Simulation of this LP solution improved lifetime by $500 \%$ over a static sink [24].

In our work we recreate the LP model as it fits to our network constraints. Using equation 2, we apply the network constraints to our shortest path routing algorithm and the network's initial node energy level. After an LP calculation of the optimal sink sojourn times, we schedule the sink to move incrementally through the network, cycling through all indicated sinks until the first time to failure.

\section{E. Dynamic Sink Movement}

Dynamic mobility patterns extend controlled mobility patterns to account for continually changing networks. Where and how the sink moves depends on things such as the changing energy of the network, whether there have been any loss 

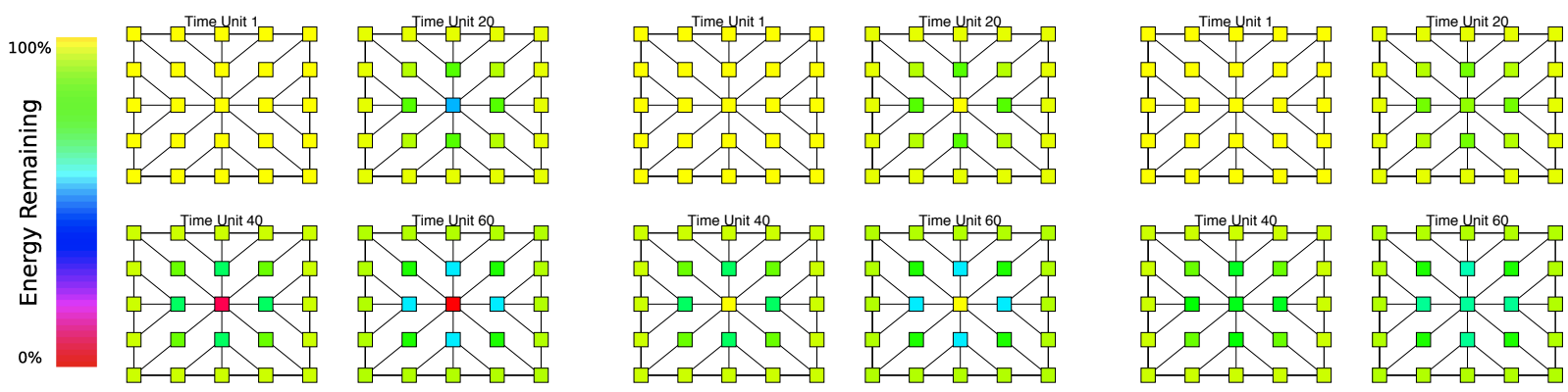

Fig. 3. Energy Drain Over Time with a Static Sink and: (a) No Recharging, (b) Sink Recharging, and (c) Lowest Node Recharging

of nodes or data packets, and any congestion problems that appear as the network is operating. Our final algorithm, Greedy Heuristic Sink, uses an approach from this category introduced in Basagni et al. [4].

This work identified a common problem in multi-hop networks in that the nodes surrounding the sink become inundated with forwarding data packets to the sink, unevenly draining these nodes of their energy. Using this observation this paper created a heuristic called Greedy Maximum Residual Energy (GMRE). The GMRE heuristic dynamically moves the sink such that it occupies the portion of the network with the highest energy levels. In simulation, the GMRE heuristic outperformed the static sink by $200 \%$ to $350 \%$ [4].

This paper takes this heuristic and applies it to a rechargeable network. As the network is operating, the sink polls all of the sensors' energy levels and compares them in blocks. Blocks within the network consist of 9 nodes, blocks near the edges of the network consist of 6 nodes, and blocks on the corners of the network consist of 4 nodes.

For each block, the algorithm calculates the energy of that block and selects the center of the largest energy block as the location of the next sink. Note that the computation of energy accounts for differing block sizes.

\section{Results}

Given our models and set of sink algorithms we now evaluate their performance and parameters. In this section, we present the simulation environment and results of the simulations. We divide results based on our three questions from before: (1) the impact of recharging on network lifetime, (2) the choice of recharging the sink or the node with least energy, and (3) the choice of sink algorithms for improved network lifetime with recharging.

\section{A. Simulation Environment}

All results were simulated in MATLAB. Each node began with a battery charge of 50 battery units and the simulation was run for a total of 200 time units. Every message that was sent or received had a cost of 0.05 battery units. We recharged nodes with a charge amount linearly proportional to the size of the network. This meant larger networks received a larger charge. Larger charge could happen in practice by sending multiple or larger UAVs), although we expect that messaging costs will go up with the square of the network size.

\section{B. Impact of Recharging on Network Lifetime}

To assess the impact of single node recharging on the life of the network, we created a five by five grid with a static sink and simulated the three types of recharging: no recharging, recharging the sink every time unit, and recharging the lowest powered node. Previous research has proven that the optimal static sink placement for optimizing network lifetime is in the center of the grid, so when conducting this research all static sink algorithms has this sink placement [1]. We then took a closer look at the effect of the power infusion, or lack thereof, at specific time units out of each simulation. Figure 3(a) shows the simulation without any recharging at four different time units. As seen in the figure, the sink and the nodes closest to the sink lose their energy quickly with the sink running out of battery near 40 time units while the perimeter nodes have most of their energy left.

Figures 3(b) and 3(c) also show the five by five grids with a static sink; however, this time the system employs a power recharging method where one node recharges every time unit. Figure 3(b) demonstrates recharging the sink, which increases network lifetime over $350 \%$ compared to no recharging, dying at time unit 144. This time it is the nodes closest to the sink that cause the network to die. The nodes along the perimeter have more of their energy drained than seen in the no recharging situation.

Figure 3(c) shows the network with recharging capabilities applied to the lowest node. This form of recharging keeps the network alive for an extra 40 time units compared to recharging the sink. In this situation, the sink and its immediate neighbors die at the same time.

These results suggest that recharging even a single node has a large impact on the network lifetime. To further explore this effect, we simulate a static sink with different sized grids and compare network lifetimes based on number of nodes in the network. Figure 4 indicates that limited recharging does improve network lifetime and that recharging the lowest node instead of the sink may provide the longest lifetime. It also demonstrates that network size has a large impact on network lifetime in the case of limited recharging. As network size increases, network lifetime decreases because the network begins to use more energy than limited recharging can supply, since the messaging growth is quadratic, while the recharging is defined as linear with the network size. In smaller grid 
sizes, adding in recharging capabilities could lead to an infinite network lifetime as long as the recharging amount is greater than the node with the heaviest routing cost.

\section{Better to Recharge Sink or Lowest Energy Node}

Figure 4 tends to indicate that recharging the lowest energy node significantly outperforms recharging the sink. We explore this and the impact of the sink selection algorithms in more detail in this section. To do so, we simulated all five sink selection and positioning algorithms with the three different forms of recharging across eighty different grid sizes ranging from 9 to 360 nodes in a grid.

All but the static algorithm intentionally move the sink to avoid the situation where the sink's neighbors limit network lifetime.

Figure 5 compares the average network lifetime of the varying grid sizes for the three forms of recharging. For most of the five sink selection algorithms, recharging the lowest node produced the longest lifetimes and no recharging produced the lowest network lifetimes. Note that we limited the number of network iterations to 200, so recharging the lowest node for LP and Greedy yielded networks that did not die over this time frame. Recharging the sink also increased network life but, in most cases, it only outperformed no recharging by a small margin. This validates our prior observation that recharging the lowest energy node is the best approach and also leads to an easy approach to maximize network life regardless of the sink selection algorithm that is being employed.

D. Best Sink Positioning Algorithm given Recharging Capabilities

Having shown that recharging the lowest energy node is optimal in all five sink positioning algorithms, we now investigate which sink positioning algorithm performs best over a wide range of network sizes using this recharging approach. Figure 6 shows the results of this comparison, comparing the network lifetime as the network size increases. The Random

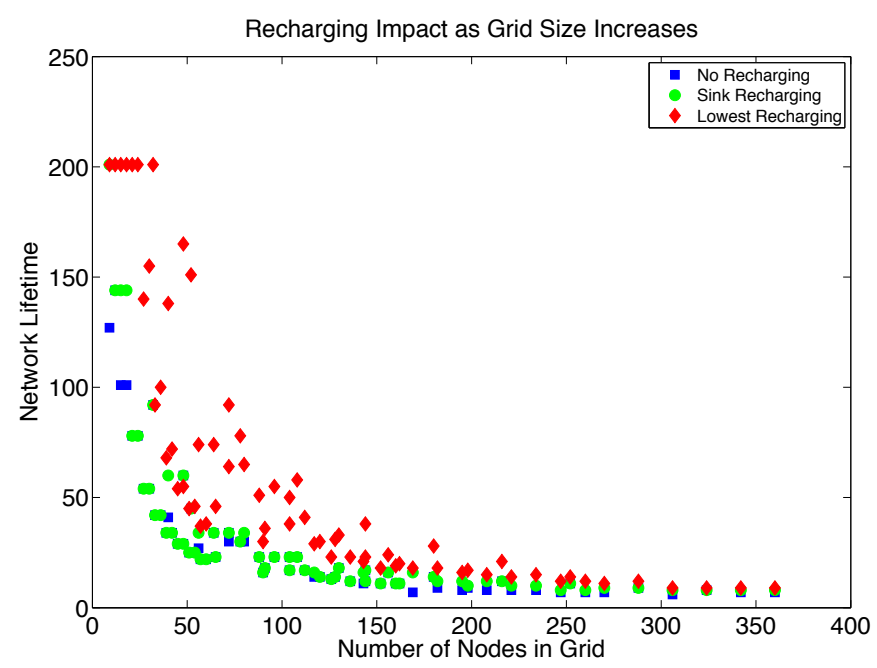

Fig. 4. All Three Types of Recharging Across Varying Grid Sizes

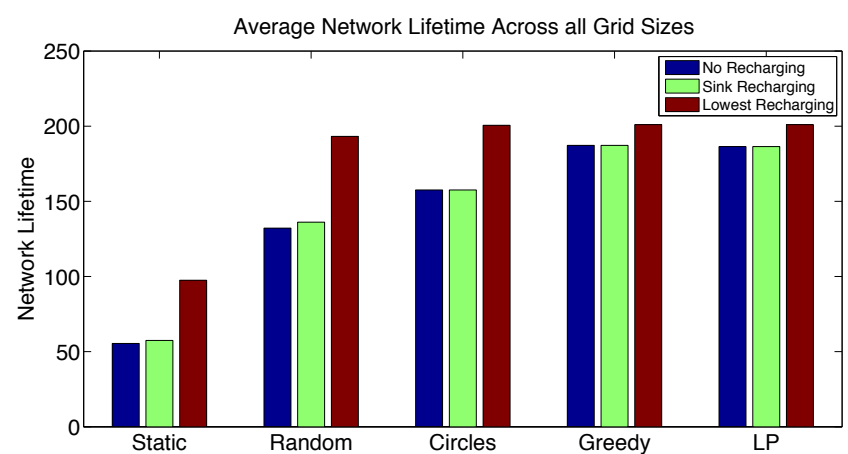

Fig. 5. All Three Types of Recharging Across All 5 Sink Positioning Algorithms

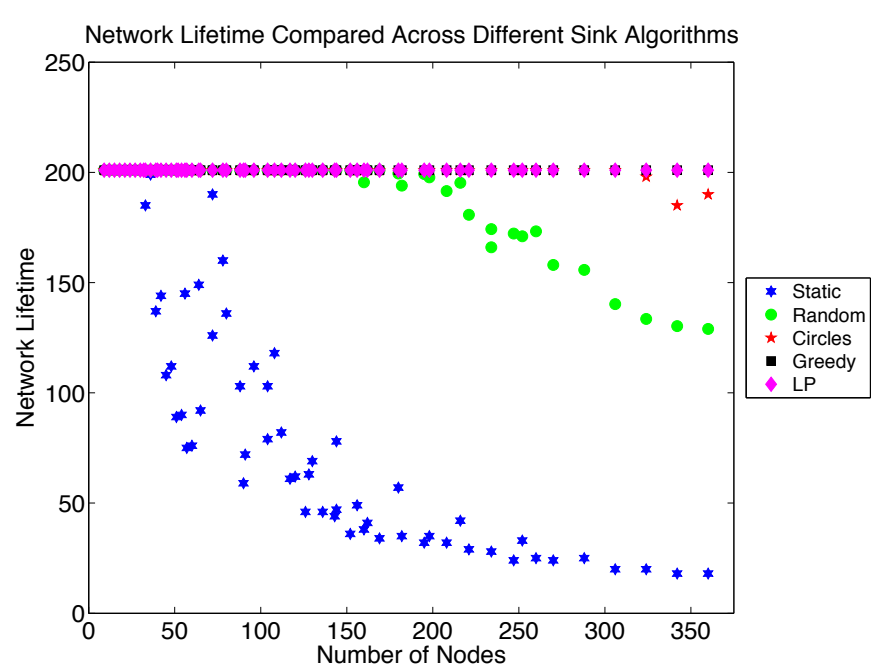

Fig. 6. All 5 Sink Positioning Algorithms with Lowest Node Recharging

Sink algorithm is a good benchmark, as the approach is easy to implement and provides the average of all possible sink selection algorithms due to its randomness. We can see that the Static Sink algorithm performs far worse than the random approach. This reinforces the poor performance that occurs when sinks do not move.

On the other hand, choosing a sink by moving along the perimeter of the network, the Circles Sink algorithm, performs well until the network size increases beyond 250 nodes at which point the network lifetime begins to decrease. The Greedy Heuristic Sink and LP Sink algorithms perform the best with no decrease in network lifetime as the network size increases. Considering that the runtime of the LP Sink algorithm is significantly higher than the Greedy Heuristic Sink algorithm, we can conclude from these investigations that the Greedy Heuristic Sink is the best approach to maximize the network lifetime in a system with limited, single-node recharging capabilities. 


\section{CONCLUSION AND FUtURE WORK}

With new capabilities that enable unmanned aerial vehicles to wirelessly recharge sensor networks it is now feasible, in certain situations, to consider limited recharging as a viable solution to the sensor network power problem. This paper explores how limited recharging of a single node affects the network lifetime with different sink selection algorithms.

Our results show that recharging the sensor network has a positive impact on the network lifetime across different grid sizes from 5 to 350 nodes. We determined that recharging the lowest powered node at every time unit proved more beneficial to the network than just recharging the sink. This result is independent of the how the sink is selected and even holds true if the sink is randomly changed at each time step.

The results examined the optimal sink positioning algorithm with the lowest powered node being recharged. It is evident that a static sink performs poorly compared to the other four algorithms. The greedy maximal residual energy heuristic and linear programming formulation performed best throughout all spans of grid sizes. Considering the drastically different runtime between the LP approach versus the greedy method, it is clear that greedy sink selection is a very good approach in networks with single node recharging capabilities.

For future work, we plan to implement a subset of these algorithms on our sensor network to verify their performance under real-world conditions. In addition, we plan to continue our simulation study to explore the effect of recharging on more complex network models including variations in topologies, packet delay, and routing congestion. We also plan to expand the scenario to explore allowing the UAV to charge more than one node as well as multiple UAVs recharging larger networks. Finally, we envision that the UAV can collect data from the network and perform data muling while recharging nodes. For this, it would be best if the UAV is in direct communication range of the sink when recharging nodes. Therefore, we are also actively exploring approaches to ensure that the sink is within range of the node being recharged.

\section{ACKNOWLEDGMENT}

We are grateful to NSF CNS (CSR-1217400 and CSR1217428) and the University of the Pacific SURF program, which partially supported this work.

\section{REFERENCES}

[1] K. Akkaya and A. Newell. Self-deployment of sensors for maximized coverage in underwater acoustic sensor networks. Computer Communications, 32(7-10):1233-1244, May 2009.

[2] Kemal Akkaya, Mohamed Younis, and Meenakshi Bangad. Sink repositioning for enhanced performance in wireless sensor networks. Computer Networks, 49(4):512-534, November 2005.

[3] P. Baruah, R. Urgaonkar, and B. Krishnamachari. Learning-enforced time domain routing to mobile sinks inwireless sensor fields. In Local Computer Networks, 2004. 29th Annual IEEE International Conference, pages 525 - 532, nov 2004.

[4] Stefano Basagni, Alessio Carosi, Emanuel Melachrinoudis, Chiara Petrioli, and Z. Wang. Controlled sink mobility for prolonging wireless sensor networks lifetime. Wireless Networks, 14(6):831-858, 2008.
[5] Ioannis Chatzigiannakis, Athanasios Kinalis, and Sotiris Nikoletseas. Efficient data propagation strategies in wireless sensor networks using a single mobile sink. Computer Communications, 31(5):896-914, March 2008.

[6] Michael Fraser, Ahmed Elgamal, Xianfei He, and Joel P. Conte. Sensor network for structural health monitoring of a highway bridge. Journal of Computing in Civil Engineering, 24(1):11-24, 2010.

[7] M. Garcia-Luna-Aceves, J.J.; Spohn. Source-tree routing in wireless networks. In Proceedings. Seventh International Conference, pages 273 282, nov 1999.

[8] B. Griffin and C. Detweiler. Resonant wireless power transfer to ground sensors from a uav. In IEEE International Conference on Robotics and Automation (ICRA), 2012.

[9] P.D. Hossein Zadeh, C. Schlegel, and M.H. MacGregor. Distributed optimal dynamic base station positioning in wireless sensor networks. Computer Networks, 56(1):34-49, January 2012.

[10] Emily Kaiser. Minnesota bridge collapse. http://minnesota.publicradio. org/collections/special/2007/bridge_collapse/. Accessed Dec. 2011.

[11] H.S. Kim, Abdelzaher, and W.H. Kwon. Minimum energy asynchronous dissemination to mobile sinks in wireless sensor networks. In Proceedings of the First Internations Conference on Embedded Networked Sensor Systems, SenSys 2003, pages 193-204, nov 2003.

[12] Sukun Kim, Shamim Pakzad, David Culler, James Demmel, Gregory Fenves, Steve Glaser, and Martin Turon. Wireless sensor networks for structural health monitoring. In Proceedings of the 4th international conference on Embedded networked sensor systems, SenSys '06, pages 427-428, New York, NY, USA, 2006. ACM.

[13] A. Ledeczi, T. Hay, P. Volgyesi, D.R. Hay, A. Nadas, and S. Jayaraman. Wireless acoustic emission sensor network for structural monitoring. Sensors Journal, IEEE, 9(11):1370-1377, nov. 2009.

[14] Luke S. Lee, Vistasp M. Karbhari, and Charles Sikorsky. Structural health monitoring of cfrp strengthened bridge decks using ambient vibrations. Structural Health Monitoring, 6(3):199-214, 2007.

[15] Jun Luo. Joint mobility and routing for lifetime elongation in wireless sensor networks. In INFOCOM 2005. 24th Annual Joint Conference of the IEEE Computer and Communications Societies. Proceedings IEEE, volume 3, pages 1735- 1746, mar 2005.

[16] Mirela Marta and Mihaela Cardei. Improved sensor network lifetime with multiple mobile sinks. Pervasive and Mobile Computing, 5(5):542_ 555, October 2009.

[17] Jeff Wise. Popular Mechanics. Engineers cite vibrations, wind in bay bridge failure. http://www.popularmechanics.com/technology/ engineering/infrastructure/4335323. Accessed Dec. 2011.

[18] D. Musiani, K. Lin, and T. Simunic Rosing. Active sensing platform for wireless structural health monitoring. In Proceedings of the 6th international conference on Information processing in sensor networks, IPSN '07, pages 390-399, New York, NY, USA, 2007. ACM.

[19] Ioannis Papadimitriou and Leonidas Georgiadis. maximum lifetime routing to mobile sink in wireless sensor networks. In Proceedings of the 2005 International Conference on Sofware, Telecommunications and Computer Networks, SoftCOM 2005, September 2005.

[20] Md Nafees Rahman and MA Matin. Efficient algorithm for prolonging network lifetime of wireless sensor networks. Tsinghua Science \& Technology, 16(6):561-568, December 2011.

[21] Ahmed Safwat, Hossam Hassanein, and Hussein Mouftah. Poweraware virtual base stations for wireless mobile ad hoc communications. Computer Networks, 41(3):331-346, February 2003.

[22] Dusan Stevanovic. Sink mobility in wireless sensor networks: Theoretical and practical considerations. M.Sc., York University (Canada), Canada, 2010.

[23] R. Vedantham, R. Sivakumar, and S.-J. Park. Sink-to-sensors congestion control. Ad Hoc Networks, 5(4):462-485, May 2007.

[24] Z.M. Wang, S. Basagni, E. Melachrinoudis, and C. Petrioli. Exploiting sink mobility for maximizing sensor networks lifetime. In Proceedings of the 38th Annual Hawaii International Conference on System Sciences, 2005. HICSS '05, page 287a, January 2005.

[25] D. Wei Ye; Heidemann, J.; Estrin. An energy-efficient mac protocol for wireless sensor networks. In INFOCOM 2002. Twenty-First Annual Joint Conference of the IEEE Computer and Communications Societies. Proceedings. IEEE, volume 3, pages 1567-1576, 2002.

[26] Jennifer Yick, Biswanath Mukherjee, and Dipak Ghosal. Wireless sensor network survey. Computer Networks, 52(12):2292-2330, August 2008

[27] Feng Zhao and Leonidas J. Guibas. Wireless sensor networks: an information processing approach. Morgan Kaufmann, 2004. 